\title{
Stabilization and trajectory tracking control for underactuated quadrotor helicopter subject to wind-gust disturbance
}

\author{
MOHD ARIFFANAN MOHD BASRI*, ABDUL RASHID HUSAIN \\ and KUMERESAN A DANAPALASINGAM
}

Department of Control and Mechatronics Engineering, Faculty of Electrical Engineering, Universiti Teknologi Malaysia, 81310 Skudai, Johor, Malaysia e-mail: ariffanan@fke.utm.my

MS received 20 April 2014; revised 2 December 2014; accepted 24 February 2015

\begin{abstract}
The control of quadrotor helicopter has been a great challenge for control engineers and researchers since quadrotor is an underactuated and a highly unstable nonlinear system. In this paper, the dynamic model of quadrotor has been derived and a so-called robust optimal backstepping control (ROBC) is designed to address its stabilization and trajectory tracking problem in the existence of external disturbances. The robust controller is achieved by incorporating a prior designed optimal backstepping control $(\mathrm{OBC})$ with a switching function. The control law design utilizes the switching function in order to attenuate the effects caused by external disturbances. In order to eliminate the chattering phenomenon, the sign function is replaced by the saturation function. A new heuristic algorithm namely Gravitational Search Algorithm (GSA) has been employed in designing the OBC. The proposed method is evaluated on a quadrotor simulation environment to demonstrate the effectiveness and merits of the theoretical development. Simulation results show that the proposed ROBC scheme can achieve favorable control performances compared to the OBC for autonomous quadrotor helicopter in the presence of external disturbances.
\end{abstract}

Keywords. Quadrotor helicopter; robust control; backstepping control; gravitational search algorithm.

\section{Introduction}

Currently, the interest for vertical take-off and landing (VTOL) vehicles is strongly increasing. This is due to their ability to hover flight and their lack of necessity for runways make them wellsuited options for supervision, inspection, and work in environments where space is limited and high maneuverability is required. The quadrotor is a four-rotor VTOL vehicle which has several advantages over traditional helicopters in terms of maneuverability, motion control and cost.

*For correspondence 
However, quadrotors are inherently unstable, nonlinear, multivariable and underactuated systems. Hence, the control of a quadrotor becomes quite a complex and difficult mainly due to its underactuated properties and nonlinearities. In literature, different studies have addressed modeling and control of underactuated nonlinear systems and variety of approaches are employed (Zilic et al 2013; Yih 2013; Xu et al 2012; Yang \& Yang 2012; Yu et al 2012).

To solve the quadrotor helicopter control problem, many techniques have been proposed such as linear quadratic regulator (LQR) control (Nuchkrua \& Parnichkun 2012; Jafari et al 2010; Castillo et al 2005), proportional-integral-derivative (PID) control (Junior et al 2013; Bolandi et al 2013; Hwang et al 2012), fuzzy logic (FL) control (Baek et al 2013; Erginer \& Altug 2012; Santos et al 2010), feedback linearization control (Zhang et al 2013; Mukherjee \& Waslander 2012; Mokhtari et al 2006), sliding mode control (Sumantri et al 2013; Guisser \& Medromi 2009; Bouadi et al 2007) and backstepping control (Lee et al 2013; Regula \& Lantos 2011; Madani \& Benallegue 2006). Initially, most of the control strategies are based on linearized models without compensation of modeling errors and external disturbances. Then, in the past few years, more efforts have been directed toward the research of dealing with uncertainties and disturbances associated with nonlinear quadrotor dynamic model.

In this paper, the control problem of the quadrotor helicopter in the presence of external disturbances is considered. The dynamical model describing the quadrotor helicopter motions and taking into account for various parameters which affect the dynamics of a flying structure is presented. Subsequently, a control strategy based on backstepping approach taking into account the external disturbances is developed. The backstepping control scheme is a nonlinear control method based on the Lyapunov theorem. The backstepping control design techniques have received great attention because of its systematic and recursive design methodology for nonlinear feedback control. The backstepping control approach has been successfully implemented in various forms to numerous real-world applications such as satellite (Kristiansen et al 2009), suspension systems (Sun et al 2013), aircraft (Shen et al 2013), chemical reactor (Bošković \& Krstić 2002), flexible spacecraft (Jiang et al 2010), robot manipulator (Hu et al 2012), electrohydraulic actuator (Kim et al 2010), and wheeled inverted pendulum systems (Chiu et al 2011). The advantage of backstepping compared with other control methods lies in its design flexibility, due to its recursive use of Lyapunov functions. The key idea of the backstepping design is to select recursively some appropriate state variables as virtual inputs for lower dimension subsystems of the overall system and the Lyapunov functions are designed for each stable virtual controller (Krstic et al 1995). Therefore, the final designed actual control law can guarantee the stability of total control system.

Although the nonlinear control realized by backstepping method can meet the desired performance of the system, it is just limited to the nominal system i.e. the system dynamics and the external disturbance are exactly known. To overcome this drawback, a nominal controller based on backstepping technique combined with a switching function is proposed to deal with the control problem of the quadrotor helicopter under the influence of external disturbances. Compared with the only backstepping control scheme (Madani \& Benallegue 2006), the present control approach has the advantages of robust control, which makes this approach attractive for a wide class of nonlinear systems with the influences of external disturbances. To solve the problem of determining the control design parameters, Gravitational Search Algorithm (GSA) has been used. Finally, the synthesized control law is highlighted by simulations which gave fairly satisfactory results despite the occurrence of external disturbances. The main contribution of this paper is a successful development of a new backstepping-based nonlinear control structure for quadrotor helicopter perturbed by external disturbances. Unlike the method presented in 
Bouadi et al (2008), the developed control scheme has the advantage of free from the chattering phenomena.

\section{Quadrotor systems modeling}

\subsection{Quadrotor description}

The quadrotor helicopter, shown in figure 1, has four rotors to generate the propeller forces $F_{i}=$ 1, 2, 3, 4. The four rotors can be thought of as two pairs, (1,3)@(front, back) and (2,4)@(left, right). One pair rotates clockwise, while the other rotates counter clockwise in order to balance the torques and produce yaw motion as needed. Yaw motion can be obtained from the difference in the counter torque between each pair of propellers, $(1,3)$ and $(2,4)$. When all four rotors are spinning with the same angular velocity the net yaw is zero, and a difference in velocities between the two pairs creates either positive or negative yaw motion. The up (down) motion can be achieved by increasing (decreasing) the rotor speeds altogether with the same magnitude. Forward (backward) motion which is related to the pitch, $\theta$ angle can be obtained by increasing the back (front) rotor thrust and decreasing the front (back) rotor thrust. Finally, a sideways motion which is related to the roll, $\phi$ angle can be achieved by increasing the left (right) rotor thrust and decreasing the right (left) rotor thrust. Figure 2 shows the various movements of a quadrotor due to changes in rotor speeds.

In order to develop the model of the quadrotor, reasonable assumptions are established in order to accommodate the controller design. The assumptions are as follows:

Assumption 1: Quadrotor is a rigid body and has symmetric structure.

Assumption 2: Aerodynamic effects can be ignored at low speed.

Assumption 3: The rotor dynamics are relatively fast and thus can be neglected.

Assumption 4: The quadrotor's center of mass and body-fixed frame origin coincide.

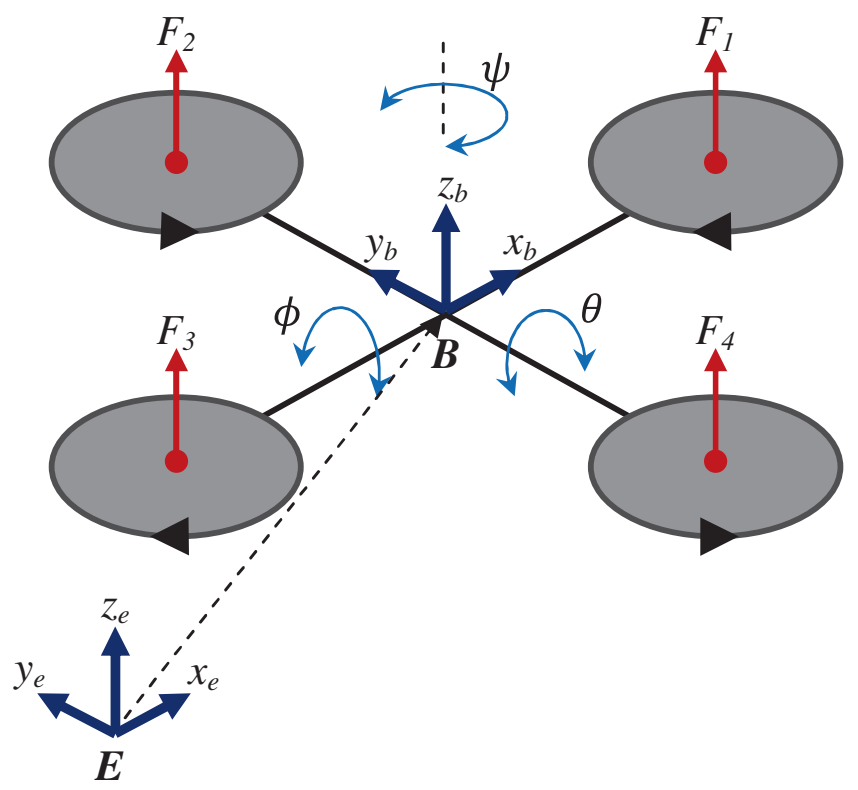

Figure 1. Quadrotor helicopter configuration. 


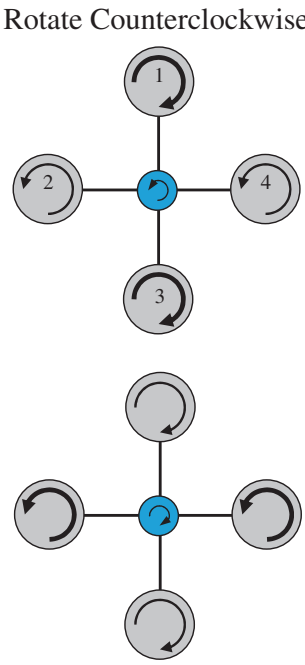

Rotate Clockwise
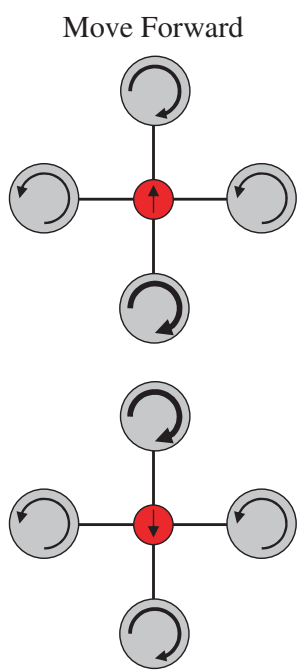

Move Backward
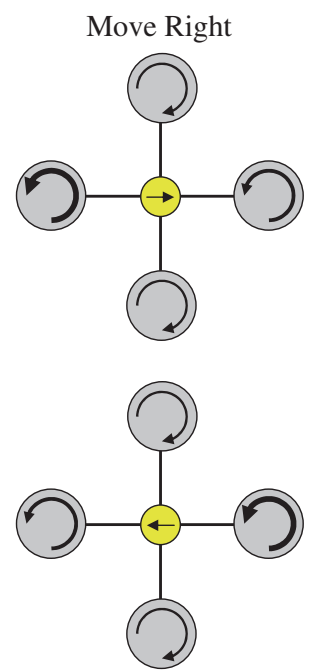

Move Left
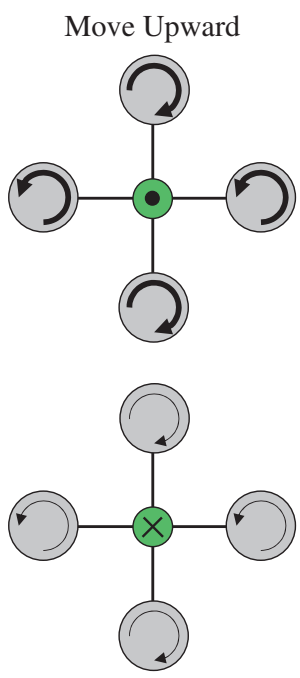

Move Downward

Figure 2. The movements of a quadrotor: the arrow width is proportional to rotor speeds.

\subsection{Quadrotor kinematic model}

Consider earth fixed frame $E=\left\{x_{e}, y_{e}, z_{e}\right\}$ and body fixed frame $B=\left\{x_{b}, y_{b}, z_{b}\right\}$, as seen in figure 1. Let $q=(x, y, z, \phi, \theta, \psi) \in R^{6}$ be the generalized coordinates for the quadrotor, where $(x, y, z)$ denote the absolute position of the rotorcraft and $(\phi, \theta, \psi)$ are the three Euler angles (roll, pitch and yaw) that describe the orientation of the aerial vehicle. Therefore, the model could be separated into two coordinate subsystems: translational and rotational. They are defined respectively by

$$
\begin{gathered}
\xi=(x, y, z) \in R^{3} \\
\eta=(\phi, \theta, \psi) \in R^{3} .
\end{gathered}
$$

The kinematic equations of the translational and rotational movements are obtained by means of the rotation $R$ and transfer $T$ matrices, respectively. The expression of the rotation $R$ and transfer $T$ matrices can be found in Olfati-Saber (2000) and defined accordingly by (3) and (4):

$$
\begin{gathered}
R=\left(\begin{array}{ccc}
c \theta c \psi & s \phi s \theta c \psi-c \phi s \psi & c \phi s \theta c \psi+s \phi s \psi \\
c \theta s \psi & s \phi s \theta s \psi+c \phi c \psi & c \phi s \theta s \psi-s \phi c \psi \\
-s \theta & s \phi c \theta & c \phi c \theta
\end{array}\right) \\
T=\left(\begin{array}{ccc}
1 & s \phi t \theta & c \phi t \theta \\
0 & c \phi & -s \phi \\
0 & s \phi / c \theta & c \phi / c \theta
\end{array}\right),
\end{gathered}
$$

where $s(\cdot), c(\cdot)$ and $t(\cdot)$ are abbreviations for $\sin (\cdot), \cos (\cdot)$ and $\tan (\cdot)$, respectively.

The translational kinematic can be written as

$$
\dot{\xi}=R V,
$$

where $\dot{\xi}$ and $V$ are, respectively, the linear velocity vector w.r.t. the earth fixed frame $E$ and body fixed frame $B$. 
The rotational kinematics can be defined as follows:

$$
\dot{\eta}=T \omega,
$$

where $\dot{\eta}$ and $\omega$ are the angular velocity vector w.r.t. the earth fixed frame $E$ and body fixed frame $B$, respectively.

\subsection{Quadrotor dynamic model}

The dynamic model of quadrotor is derived from Newton-Euler approach. It can be useful to express the translational dynamic equations w.r.t. the earth fixed frame $E$ and rotational dynamic equations w.r.t. the body fixed frame $B$.

Therefore, the translational dynamic equations of quadrotor can be written as follows:

$$
m \dot{\xi}=-m g e_{z}+u_{T} R e_{z},
$$

where $m$ denotes the quadrotor mass, $g$ the gravity acceleration, $e_{z}=(0,0,1)^{T}$ the unit vector expressed in the frame $E$ and $u_{T}$ the total thrust produced by the four rotors.

$$
u_{T}=\sum_{i=1}^{4} F_{i}=b \sum_{i=1}^{4} \Omega_{i}^{2},
$$

where $F_{i}$ and $\Omega_{i}$ denote respectively, the thrust force and speed of the rotor $i$ and $b$ is the thrust factor.

The rotational dynamic equations of quadrotor can be written as follows:

$$
I \dot{\omega}=-\omega \times I \omega-G_{a}+\tau,
$$

where $I$ is the inertia matrix, $-\omega \times I \omega$ and $G_{a}$ are the gyroscopic effect due to rigid body rotation and propeller orientation change respectively, while $\tau$ is the control torque obtained by varying the rotor speeds. $G_{a}$ and $\tau$ are defined as

$$
\begin{gathered}
G_{a}=\sum_{i=1}^{4} J_{r}\left(\omega \times e_{z}\right)(-1)^{i+1} \Omega_{i} \\
\tau=\left(\begin{array}{c}
\tau_{\phi} \\
\tau_{\theta} \\
\tau_{\psi}
\end{array}\right)=\left(\begin{array}{c}
l b\left(\Omega_{4}^{2}-\Omega_{2}^{2}\right) \\
l b\left(\Omega_{3}^{2}-\Omega_{1}^{2}\right) \\
d\left(\Omega_{2}^{2}+\Omega_{4}^{2}-\Omega_{1}^{2}-\Omega_{3}^{2}\right)
\end{array}\right),
\end{gathered}
$$

where $J_{r}$ is the rotor inertia, $l$ represents the distance from the rotors to the centre of mass and $d$ is the drag factor.

Then, by recalling (7) and (9), the dynamic model of the quadrotor in terms of position $(x, y, z)$ and rotation $(\phi, \theta, \psi)$ is written as

$$
\begin{gathered}
\left(\begin{array}{c}
\ddot{x} \\
\ddot{y} \\
\ddot{z}
\end{array}\right)=\left(\begin{array}{c}
0 \\
0 \\
-g
\end{array}\right)+\frac{1}{m}\left(\begin{array}{c}
c_{\phi} s_{\theta} c_{\psi}+s_{\phi} s_{\psi} \\
c_{\phi} s_{\theta} s_{\psi}-s_{\phi} c_{\psi} \\
c_{\phi} c_{\theta}
\end{array}\right) u_{T} \\
\left(\begin{array}{c}
\ddot{\phi} \\
\ddot{\theta} \\
\ddot{\psi}
\end{array}\right)=\left(\begin{array}{c}
\dot{\theta} \dot{\psi}\left(\frac{I_{y y}-I_{z z}}{I_{x x}}\right) \\
\dot{\phi} \dot{\psi}\left(\frac{I_{z z}-I_{x x}}{I_{y y}}\right) \\
\dot{\theta} \dot{\phi}\left(\frac{I_{x x}-I_{y y}}{I_{z z}}\right)
\end{array}\right)-\left(\begin{array}{c}
\frac{J_{r}}{I_{x x}} \dot{\theta} \Omega_{d} \\
-\frac{J_{r}}{I_{y y}} \dot{\phi} \Omega_{d} \\
0
\end{array}\right)+\left(\begin{array}{c}
\frac{1}{I_{x x}} \tau_{\phi} \\
\frac{1}{I_{y y}} \tau_{\theta} \\
\frac{1}{I_{z z}} \tau_{\psi}
\end{array}\right) .
\end{gathered}
$$


Consequently, quadrotor is an underactuated system with six outputs $(x, y, z, \phi, \theta, \psi)$ and four control inputs $\left(u_{T}, \tau_{\phi}, \tau_{\theta}, \tau_{\psi}\right)$.

Finally, the quadrotor dynamic model can be written in the following form:

$$
\begin{aligned}
\ddot{x} & =\left(c_{\phi} s_{\theta} c_{\psi}+s_{\phi} s_{\psi}\right) \frac{1}{m} u_{1} \\
\ddot{y} & =\left(c_{\phi} s_{\theta} s_{\psi}-s_{\phi} c_{\psi}\right) \frac{1}{m} u_{1} \\
\ddot{z} & =-g+\left(c_{\phi} c_{\theta}\right) \frac{1}{m} u_{1} \\
\ddot{\phi} & =\dot{\theta} \dot{\psi}\left(\frac{I_{y y}-I_{z z}}{I_{x x}}\right)-\frac{J_{r}}{I_{x x}} \dot{\theta} \Omega_{d}+\frac{l}{I_{x x}} u_{2} \\
\ddot{\theta} & =\dot{\phi} \dot{\psi}\left(\frac{I_{z z}-I_{x x}}{I_{y y}}\right)+\frac{J_{r}}{I_{y y}} \dot{\phi} \Omega_{d}+\frac{l}{I_{y y}} u_{3} \\
\ddot{\psi} & =\dot{\theta} \dot{\phi}\left(\frac{I_{x x}-I_{y y}}{I_{z z}}\right)+\frac{1}{I_{z z}} u_{4}
\end{aligned}
$$

with a renaming of the control inputs as

$$
\begin{aligned}
& u_{1}=b\left(\Omega_{1}^{2}+\Omega_{2}^{2}+\Omega_{3}^{2}+\Omega_{4}^{2}\right) \\
& u_{2}=b\left(\Omega_{4}^{2}-\Omega_{2}^{2}\right) \\
& u_{3}=b\left(\Omega_{3}^{2}-\Omega_{1}^{2}\right) \\
& u_{4}=d\left(\Omega_{2}^{2}+\Omega_{4}^{2}-\Omega_{1}^{2}-\Omega_{3}^{2}\right)
\end{aligned}
$$

and the $\Omega_{d}$ is formulated as

$$
\Omega_{d}=\Omega_{2}+\Omega_{4}-\Omega_{1}-\Omega_{3}
$$

\section{Backstepping control system for quadrotor}

The dynamic model (14) with the consideration of external disturbance can be represented into nonlinear dynamic equation described as follows:

$$
\ddot{X}=f(X)+g(X) u+\delta,
$$

where $u, X$ and $\delta$ are respectively the input, state and external disturbance vector given as follows:

$$
\begin{aligned}
& u=\left[\begin{array}{llll}
u_{1} & u_{2} & u_{3} & u_{4}
\end{array}\right]^{T} \\
& X=\left[\begin{array}{llllll}
x_{1} & x_{2} & x_{3} & x_{4} & x_{5} & x_{6}
\end{array}\right]^{T}=\left[\begin{array}{lllll}
z & \phi & \psi & \psi & x
\end{array}\right]^{T} \\
& \delta=\left[\begin{array}{llllll}
\delta_{1} & \delta_{2} & \delta_{3} & \delta_{4} & \delta_{5} & \delta_{6}
\end{array}\right]^{T} .
\end{aligned}
$$


The bound of the external disturbance is assumed to be given, that is $|\delta| \leq \beta$, where $\beta$ is a given positive constant. From (14) and (19), the nonlinear dynamic function $f(X)$ and nonlinear control function $g(X)$ matrices can be written accordingly as

$$
f(X)=\left(\begin{array}{c}
-g \\
\dot{\theta} \dot{\psi} a_{1}-\dot{\theta} a_{2} \Omega_{d} \\
\dot{\phi} \dot{\psi} a_{3}+\dot{\phi} a_{4} \Omega_{d} \\
\dot{\theta} \dot{\phi} a_{5} \\
0 \\
0
\end{array}\right) \quad g(X)=\left(\begin{array}{cccc}
u_{z} \frac{1}{m} & 0 & 0 & 0 \\
0 & b_{1} & 0 & 0 \\
0 & 0 & b_{2} & 0 \\
0 & 0 & 0 & b_{3} \\
u_{x} \frac{1}{m} & 0 & 0 & 0 \\
u_{y} \frac{1}{m} & 0 & 0 & 0
\end{array}\right)
$$

with the abbreviations $a_{1}=\left(I_{y y}-I_{z z}\right) / I_{x x}, a_{2}=J_{r} / I_{x x}, a_{3}=\left(I_{z z}-I_{x x}\right) / I_{y y}, a_{4}=J_{r} / I_{y y}$, $a_{5}=\left(I_{x x}-I_{y y}\right) / I_{z z}, b_{1}=l / I_{x x}, b_{2}=l / I_{y y}, b_{3}=1 / I_{z z}, u_{z}=\left(c_{\phi} c_{\theta}\right)$

The control objective is to design a suitable control law for the system (17) so that the state

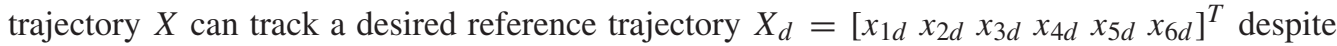
the presence of external disturbance. Since the description of the control system design of the helicopter is similar for each DOF, for simplicity only one of the six DOF is considered.

The design of ideal backstepping control (IBC) is described step-by-step as follows:

Step 1: Define the tracking error:

$$
e_{1}=x_{1 d}-x_{1}
$$

where $x_{1 d}$ is a desired trajectory specified by a reference model. Then the derivative of tracking error can be represented as

$$
\dot{e}=\dot{x}_{1 d}-\dot{x}_{1}
$$

The first Lyapunov function is chosen as

$$
V_{1}\left(e_{1}\right)=\frac{1}{2} e_{1}^{2}
$$

The derivative of $V_{1}$ is

$$
\dot{V}_{1}\left(e_{1}\right)=e_{1} \dot{e}_{1}=e_{1}\left(\dot{x}_{1 d}-\dot{x}_{1}\right)
$$

$\dot{x}_{1}$ can be viewed as a virtual control. The desired value of virtual control known as a stabilizing function can be defined as follows:

$$
\alpha_{1}=\dot{x}_{1 d}+k_{1} e_{1},
$$

where $k_{1}$ is a positive constant and should be determined by the GSA.

By substituting the virtual control by its desired value, Eq. (25) then becomes

$$
\dot{V}_{1}\left(e_{1}\right)=-k_{1} e_{1}^{2} \leq 0 \text {. }
$$

Step 2: The deviation of the virtual control from its desired value can be defined as

$$
e_{2}=\dot{x}_{1}-\alpha_{1}=\dot{x}_{1}-\dot{x}_{1 d}-k_{1} e_{1} .
$$


The derivative of $e_{2}$ is expressed as

$$
\begin{aligned}
\dot{e} & =\ddot{x}_{1}-\dot{\alpha}_{1} \\
& =f\left(x_{1}\right)+g\left(x_{1}\right) u_{1}+\delta_{1}-\ddot{x}_{1 d}-k_{1} e_{1} .
\end{aligned}
$$

The second Lyapunov function is chosen as

$$
V_{2}\left(e_{1}, e_{2}\right)=\frac{1}{2} e_{1}^{2}+\frac{1}{2} e_{2}^{2}
$$

Finding derivative of (30) yields

$$
\begin{aligned}
\dot{V}_{2}\left(e_{1}, e_{2}\right) & =e_{1} \dot{e}_{1}+e_{2} \dot{e}_{2} \\
& =e_{1}\left(\dot{x}_{1 d}-\dot{x}_{1}\right)+e_{2}\left(\ddot{x}_{1}-\dot{\alpha}_{1}\right) \\
& =e_{1}\left(-e_{2}-k_{1} e_{1}\right)+e_{2}\left(f\left(x_{2}\right)+g\left(x_{1}\right) u_{1}+\delta_{1}-\ddot{x}_{1 d}-k_{1} \dot{e}_{1}\right) \\
& =-k_{1} e_{1}^{2}+e_{2}\left(-e_{1}+f\left(x_{1}\right)+g\left(x_{1}\right) u_{1}+\delta_{1}-\ddot{x}_{1 d}-\ddot{x}_{d}-k_{1} \dot{e}_{1}\right) .
\end{aligned}
$$

Step 3: Assuming the external disturbance is well known, an IBC can be obtained as

$$
u_{1(I B)}=\frac{1}{g\left(x_{1}\right)}\left(e_{1}+k_{1} \dot{e}_{1}+\ddot{x}_{1 d}-f\left(x_{1}\right)-\delta_{1}-k_{2} e_{2}\right),
$$

where $k_{2}$ is a positive constant and should be also determined by the GSA. The term $k_{2} e_{2}$ is added to stabilize the tracking error $e_{1}$.

Substituting (32) into (31), the following equation can be obtained:

$$
\dot{V}_{2}\left(e_{1}, e_{2}\right)=-k_{1} e_{1}^{2}-k_{2} e_{2}^{2}=-E^{T} K E \leq 0,
$$

where $E=\left[e_{1} e_{2}\right]^{T}$ and $K=\operatorname{diag}\left(k_{1}, k_{2}\right)$. Since $\dot{V}_{2}\left(e_{1}, e_{2}\right) \leq 0, \dot{V}_{2}\left(e_{1}, e_{2}\right)$ is negative semi-definite.

Therefore, the IBC in (32) will asymptotically stabilize the system.

\section{Backstepping control parameters optimization}

\subsection{Overview of gravitational search algorithm}

The gravitational search algorithm (GSA) is one of the newest heuristic algorithms inspired by the Newtonian laws of gravity and motion developed by Rashedi et al (2009). In this algorithm, agents are considered as objects and their performance is measured by their masses. All these objects attract each other by the gravity force, and this force causes a global movement of all objects towards the objects with heavier masses. The heavy masses correspond to good solutions and move more slowly than lighter ones representing worse solutions. The position of the mass corresponds to a solution of the problem, and its gravitational and inertial masses are determined using a fitness function.

Consider an optimization problem of $d$ dimension and $N$ agents (masses). The position of the $i$ th agent is defined as follows:

$$
X_{i}=\left(x_{i}^{1}, x_{i}^{2}, \ldots, x_{i}^{d}\right) \text { for } i=1,2, \ldots, N .
$$

The gravitational force between agents $i$ and $j$ at time $t$ is represented as follows:

$$
F_{i j}^{d}(t)=G(t) \frac{M_{i}(t) \times M_{j}(t)}{R_{i j}(t)+\varepsilon}\left(x_{j}^{d}(t)-x_{i}^{d}(t)\right),
$$


where $M_{i}$ is the mass of the agent $i, M_{j}$ is the mass of the agent $j, G(t)$ the gravitational constant at time $t, \varepsilon$ a small constant and $R_{i j}(t)$ is the Euclidian distance between agent $i$ and $j$ objects defined as follows:

$$
R_{i j}(t)=\left\|X_{i}(t), X_{j}(t)\right\|_{2}
$$

The gravitational constant, $G$ is initialized at the beginning and will be reduced with time to control the search accuracy. In other words, $G$ is a function of the initial value $G_{0}$ and time $t$ given as follows:

$$
G(t)=G\left(G_{0}, t\right) .
$$

The total force acting on the $i$ th agent is calculated as follows:

$$
F_{i}^{d}(t)=\sum_{j=1, j \neq 1}^{N} \operatorname{rand}_{j} F_{i j}^{d}(t),
$$

where rand $_{j}$ is a random number in the interval $[0,1]$.

According to Newton's law of motion, the acceleration of the agent $i$ at time $t$ in the $d$ dimension is given as follows:

$$
a_{i}^{d}(t)=\frac{F_{i}^{d}(t)}{M_{i}(t)},
$$

where

$$
M_{i}(t)=\frac{m_{i}(t)}{\sum_{j=1}^{N} m_{j}(t)}
$$

and

$$
m_{i}(t)=\frac{\operatorname{fit}_{i}(t)-\operatorname{worst}(t)}{\operatorname{best}(t)-\operatorname{worst}(t)}
$$

where $f i t_{i}(t)$ represents the fitness value of the agent $i$ at time $t$, best $(t)$ and $\operatorname{worst}(t)$ are respectively the best fitness value and the worst fitness value of agent $i$ at time $t$.

It is to be noted that for a minimization problem:

$$
\begin{aligned}
\operatorname{best}(t) & =\min \left(f i t_{i}(t)\right) \quad i=1,2, \ldots, N \\
\operatorname{worst}(t) & =\max \left(f i t_{i}(t)\right) \quad i=1,2, \ldots, N .
\end{aligned}
$$

The updated velocity of an agent is defined as a function of its current velocity added to its current acceleration. Hence, the next position and next velocity of an agent can be formulated as follows:

$$
\begin{aligned}
& v_{i}^{d}(t+1)=\operatorname{rand}_{i} \times v_{i}^{d}(t)+a_{i}^{d}(t) \\
& x_{i}^{d}(t+1)=x_{i}^{d}(t)+v_{i}^{d}(t+1) .
\end{aligned}
$$

where $v_{i}^{d}$ and $x_{i}^{d}(t)$ are the velocity and position of an agent at $t$ time in $d$ dimension, respectively. rand $_{i}$ is a random number in the interval $[0,1]$. 


\subsection{Optimal backstepping control system}

The dynamic model in (14) can be divided into two subsystems $\Pi_{1}$ and $\Pi_{2}$, listed as follows:

$$
\begin{gathered}
\Pi_{1}:\left\{\begin{array}{l}
\ddot{\phi}=\dot{\theta} \dot{\psi}\left(\frac{I_{y y}-I_{z z}}{I_{x x}}\right)-\frac{J_{r}}{I_{x x}} \dot{\theta} \Omega_{d}+\frac{l}{I_{x x}} u_{2} \\
\ddot{\theta}=\dot{\phi} \dot{\psi}\left(\frac{I_{z z}-I_{x x}}{I_{y y}}\right)+\frac{J_{r}}{I_{y y}} \dot{\phi} \Omega_{d}+\frac{l}{I_{y y}} u_{3} \\
\ddot{\psi}=\dot{\theta} \dot{\phi}\left(\frac{I_{x x}-I_{y y}}{I_{z z}}\right)+\frac{l}{I_{z z}} u_{4}
\end{array}\right. \\
\Pi_{2}:\left\{\begin{array}{l}
\ddot{x}=(c \phi s \theta c \psi+s \phi s \psi) \frac{1}{m} u_{1} \\
\ddot{y}=(c \phi s \theta s \psi-s \phi c \psi) \frac{1}{m} u_{1} . \\
\ddot{z}=-g+(c \phi c \theta) \frac{1}{m} u_{1}
\end{array}\right.
\end{gathered}
$$

$\Pi_{1}$ in (46) represents the rotation subsystem related to the dynamics of quadrotor roll motion $\phi$, pitch motion $\theta$ and yaw motion $\psi . \Pi_{2}$ in (47) represents the position subsystem related to the dynamics of quadrotor longitude motion $x$, latitude motion $y$ and altitude motion $z$. Hence, the control scheme advocated for the overall system is then logically divided into a rotation controller and a position controller. In the previous section a controller (32) has been designed to stabilize one DOF of the overall system. The coefficients $k_{1}, k_{2}$ are control parameters and need to be positive to satisfy stability criteria. In conventional backstepping method, these parameters are selected by trial and error. It is also possible that the parameters are properly chosen, but it cannot be said that the optimal parameters are selected. To overcome this drawback, this paper adopts the GSA for determining the optimal value of the backstepping control parameters. The GSA is utilized off line to determine the backstepping controller parameters. The performance of the controller varies according to adjusted parameters. Since the optimal backstepping control (OBC) aims to improve the control performance yielded by a backstepping controller, it keeps the simple structure of the backstepping controller. As aforementioned, each of the rotation and position subsystem is comprised of three DOF. Then there are in sum six control parameters that need to be selected simultaneously for each subsystem.

In the present study, an integral absolute error $(I A E)$ is utilized to judge the performance of the controller. $I A E$ criterion is widely adopted to evaluate the dynamic performance of the control system. The index IAE is expressed as follows (De Moura Oliveira et al 2013):

$$
I A E=\int_{0}^{t}|e(t)| d t .
$$

Since the system is comprised of two subsystems, a vector integral absolute error for the rotation subsystem is taken as $I A E_{R}=\left[\begin{array}{lll}I A E_{\phi} & I A E_{\theta} & I A E_{\psi}\end{array}\right]$, where the subscripts are denoted for roll, pitch and yaw, respectively. Meanwhile, a vector integral absolute error for the position subsystem is taken as $I A E_{p}=\left[\begin{array}{lll}I A E_{x} & I A E_{y} & I A E_{z}\end{array}\right]$, where the subscripts are denoted for longitude, latitude and altitude, respectively.

For the rotation controller, GSA is utilized to minimize the fitness function $J_{R}$, expressed as

$$
J_{R}=I A E_{R} \cdot W
$$

and for the position controller, GSA is utilized to minimize the fitness function $J_{P}$, expressed as

$$
J_{P}=I A E_{P} \cdot W
$$


where $W=\left[\begin{array}{lll}W_{1} & W_{2} & W_{3}\end{array}\right]^{T}$ is weighting vector used to set the priority of the multiple objective performance index (MOPI) parameters and the value of " $W$ " varies from 0 to 1 . In this case, equal weights for the three objectives to be met by the each controller are considered as such the minimizations of the error indexes are equally important. For fitness function calculation, the time-domain simulation of the quadrotor system model is carried out for the simulation period, $t$. It is aimed to minimize this fitness function in order to improve the system response in terms of the steady-state errors.

\section{Robust optimal backstepping control system}

However, if unpredictable perturbations from the external disturbance occur, the optimized IBC (32) effort cannot ensure the favorable control performance. Thus, auxiliary control effort should be designed as switching control effort to eliminate the effect of the unpredictable perturbations. The auxiliary control effort is referred as switching control effort defined as follows:

$$
u_{1(s w)}=\lambda \operatorname{sign}\left(e_{2}\right)
$$

where $\lambda$ is a constant determined by design parameter and $\operatorname{sign}\left(e_{2}\right)$ is a sign function:

$$
\operatorname{sign}\left(e_{2}\right)=\left\{\begin{array}{cc}
1, & e_{2} / \rho>0 \\
-1, & e_{2} / \rho<0
\end{array}\right.
$$

The backstepping control effort for the nominal model $(\delta=0)$ is formulated as follows:

$$
u_{1(B)}=\frac{1}{g\left(x_{1}\right)}\left(e_{1}+k_{1} \dot{e}_{1}+\ddot{x}_{1 d}-f\left(x_{1}\right)-k_{2} e_{2}\right) .
$$

Totally, the robust optimal backstepping control (ROBC) law for one DOF of quadrotor nonlinear systems with the present of external disturbance, which guarantees the stability and convergence can be generally represented as

$$
\begin{aligned}
u_{i} & =u_{i(B)}+u_{i(s w)} \\
u_{1} & =\frac{1}{g\left(x_{1}\right)}\left(e_{1}+k_{1} \dot{e}_{1}+\ddot{x}_{1 d}-f\left(x_{1}\right)-k_{2} e_{2}\right)+\frac{\varepsilon}{g\left(x_{1}\right)} \operatorname{sign}\left(e_{2}\right) ; \quad(\text { for } i=1),
\end{aligned}
$$

where $\varepsilon$ is design parameter to be determined later.

The utilization of discontinuous switching function will excite undesired phenomenon called chatter. In order to reduce the chattering phenomenon, the most common method is to utilize the saturation function $\operatorname{sat}\left(e_{2} / \rho\right)$. Thus, replacing $\operatorname{sign}\left(e_{2}\right)$ by $\operatorname{sat}\left(e_{2} / \rho\right)$ in $(54)$ implies

$$
u_{1}=\frac{1}{g\left(x_{1}\right)}\left(e_{1}+k_{1} \dot{e}_{1}+\ddot{x}_{1 d}-f\left(x_{1}\right)-k_{2} e_{2}+\varepsilon s a t\left(e_{2} / \rho\right)\right) .
$$

The saturation function sat $\left(e_{2} / \rho\right)$ is defined as follows:

$$
\operatorname{sat}\left(e_{2} / \rho\right)=\left\{\begin{array}{rl}
\operatorname{sign}\left(e_{2} / \rho\right), & \left|e_{2} / \rho\right|>1 \\
e_{2} / \rho, & \left|e_{2} / \rho\right| \leq 1
\end{array} \Leftrightarrow \operatorname{sat}\left(e_{2} / \rho\right)=\left\{\begin{array}{cl}
1, & e_{2} / \rho>1 \\
-1, & e_{2} / \rho<-1 \\
e_{2} / \rho, & \left|e_{2} / \rho\right| \leq 1
\end{array}\right.\right.
$$

where $\rho$ is a small positive constant. 


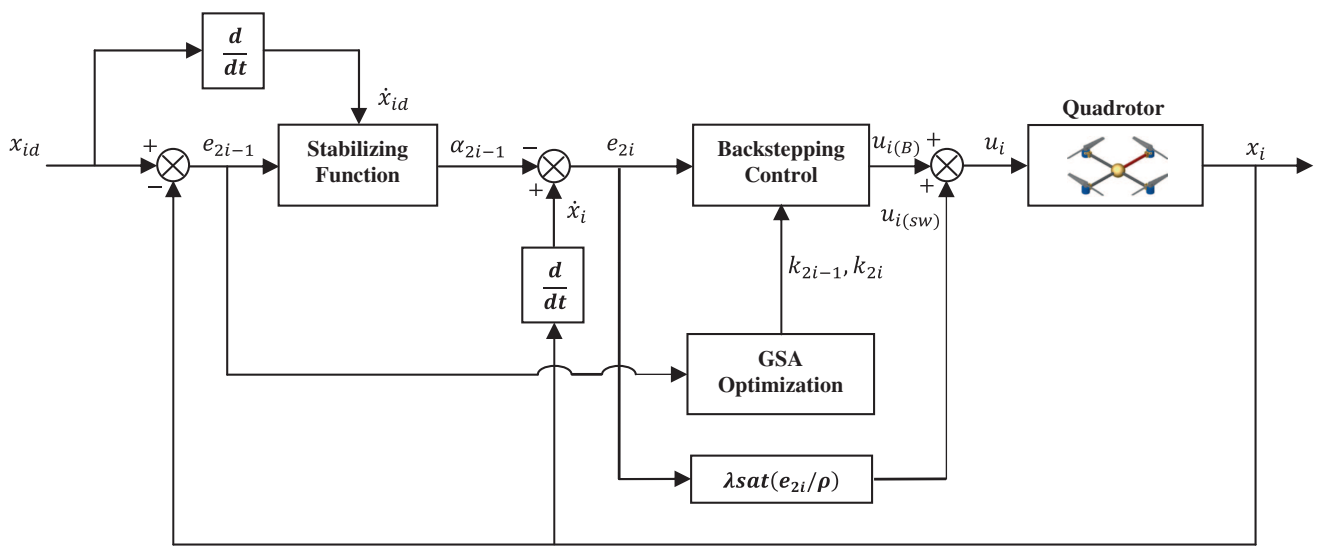

Figure 3. Block diagram of the robust optimal backstepping control system.

Theorem 1. For the nonlinear dynamic equation of quadrotor with external disturbance as represented by (17), if the control law in (55) is applied, the system will be asymptotically stable.

Proof. Consider the following Lyapunov function:

$$
V=\frac{1}{2} e_{1}^{2}+\frac{1}{2} e_{2}^{2}
$$

Differentiating (57) with respect to time leads to

$$
\begin{aligned}
\dot{V} & =e_{1} \dot{e}_{1}+e_{2} \dot{e}_{2} \\
& =e_{1}\left(-e_{2}-k_{1} e_{1}\right)+e_{2}\left(f\left(x_{1}\right)+g\left(x_{1}\right) u_{1}+\delta_{1}-\ddot{x}_{1 d}-k_{1} \dot{e}_{1}\right) \\
& =-k_{1} e_{1}^{2}+e_{2}\left(-e_{1}+f\left(x_{1}\right)+g\left(x_{1}\right) u_{1}+\delta_{1}-\ddot{X}_{1 d}-k_{1} \dot{e}_{1}\right) .
\end{aligned}
$$

Substituting the ROBC law from (55), then (58) becomes

$$
\begin{aligned}
\dot{V} & =-k_{1} e_{1}^{2}-k_{2} e_{2}^{2}+e_{2}\left(\varepsilon \operatorname{sat}\left(e_{2} / \rho\right)+\delta_{1}\right) \\
& =-k_{1} e_{1}^{2}-k_{2} e_{2}^{2}+e_{2}+\varepsilon\left|e_{2}\right|+\delta_{1} e_{2} .
\end{aligned}
$$

From Lyapunov theorem, the system will be asymptotically stable if $\dot{V}$ is negative definite. Hence, the design parameter should be chosen in such a way that $\dot{V}<0$ is always satisfied. As aforementioned, it is assumed that $\delta$ is bounded with $\beta$. So, by choosing $-\varepsilon \geq \beta$ then $\dot{V}<0$ can be guaranteed.

The configuration of the proposed control system is depicted in figure 3 .

\section{Simulation results}

In this section, the performance of the proposed approach is evaluated. The corresponding algorithm is implemented in MATLAB/SIMULINK simulation environment. The quadrotor system is modeled in SIMULINK and the GSA is implemented in MATLAB. The model parameter values of the quadrotor system are taken from Voos (2009) and listed in table 1. Initially, the controller parameter optimization is searched with the quadrotor control model, and later the 
Table 1. Parameters of the quadrotor.

\begin{tabular}{llll}
\hline Parameter & Description & Value & Units \\
\hline$g$ & Gravity & 9.81 & $\mathrm{~m} / \mathrm{s}^{2}$ \\
$m$ & Mass & 0.5 & $\mathrm{~kg}$ \\
$l$ & Distance & 0.2 & $\mathrm{~m}$ \\
$I_{x x}$ & Roll inertia & $4.85 \times 10^{-3}$ & $\mathrm{~kg} \cdot \mathrm{m}^{2}$ \\
$I_{y y}$ & Pitch inertia & $4.85 \times 10^{-3}$ & $\mathrm{~kg} \cdot \mathrm{m}^{2}$ \\
$I_{z z}$ & Yaw inertia & $8.81 \times 10^{-3}$ & $\mathrm{~kg} \cdot \mathrm{m}^{2}$ \\
$b$ & Thrust factor & $2.92 \times 10^{-6}$ & \\
$d$ & Drag factor & $1.12 \times 10^{-7}$ & \\
\hline
\end{tabular}

identified parameter values are transferred to the controller in the quadrotor system developed in MATLAB/SIMULINK for further evaluation.

In this study, the following values are assigned for controller parameters optimization:

(i) Dimension of the search space $=6$ (i.e., $k_{i=1 \ldots 6}$ or $k_{i=7 \ldots 12}$ );

(ii) The number of agents (masses) $=15$;

(iii) The number of maximum iteration $=20$;

(iv) The searching ranges for the backstepping parameters are limited to $[0,15]$;

(v) The simulation time, $t$ is equal to $10 \mathrm{~s}$;

(vi) Optimization process is repeated for 20 times.

The finest set of values among the simulation runs is selected as the best optimized controller value. The parameter and fitness values of each particle during the simulation for the rotation and position controller are summarized in tables 2 and 3, respectively. For the rotation controller, the best fitness value is $4.933 e-008$ appeared in iteration number 11, and the optimal parameters are $k_{1}=14.00, k_{2}=14.47, k_{3}=15.00, k_{4}=14.27, k_{5}=14.29$ and $k_{6}=14.62$. The variation

Table 2. The rotation controller parameters and fitness value of each optimal particle.

\begin{tabular}{lcc}
\hline Iteration no. & Optimal parameters & Fitness value \\
\hline 1 & $k_{1}=12.22, k_{2}=14.27$ & $-4.503 e-006$ \\
$k_{3}=14.47, k_{4}=14.82$ & \\
$k_{5}=14.10, k_{6}=14.13$ & \\
$k_{1}=13.65, k_{2}=12.94$ & $-3.974 e-006$ \\
$k_{3}=14.98, k_{4}=14.78$ & \\
$k_{5}=12.27, k_{6}=14.27$ & \\
& $k_{1}=12.00, k_{2}=12.00$ & \\
& $k_{3}=15.00, k_{4}=14.52$ & \\
6 & $k_{5}=13.65, k_{6}=14.47$ & \\
& $k_{1}=14.00, k_{2}=14.47$ & \\
& $k_{3}=15.00, k_{4}=14.27$ & \\
& $k_{5}=14.29, k_{6}=14.62$ & $-4.933 e-007$ \\
& $k_{1}=14.00, k_{2}=14.47$ & \\
& $k_{3}=15.00, k_{4}=14.27$ & \\
& $k_{5}=14.29, k_{6}=14.62$ & \\
\end{tabular}


Table 3. The position controller parameters and fitness value of each optimal particle.

\begin{tabular}{lcc}
\hline Iteration no. & Optimal parameters & Fitness value \\
\hline 1 & $k_{7}=14.66, k_{8}=13.88$ & 0.2476 \\
$k_{9}=13.40, k_{10}=13.14$ & \\
$k_{11}=13.37, k_{12}=14.33$ & \\
$k_{7}=15.00, k_{8}=13.77$ & 0.2072 \\
2 & $k_{9}=13.64, k_{10}=14.04$ & \\
& $k_{11}=13.77, k_{12}=15.00$ & \\
& $k_{7}=15.00, k_{8}=14.99$ & \\
& $k_{9}=13.83, k_{10}=14.41$ & \\
4 & $k_{11}=14.34, k_{12}=15.00$ & \\
& $k_{7}=15.00, k_{8}=15.00$ & \\
& $k_{9}=14.29, k_{10}=14.79$ & \\
7 & $k_{11}=14.70, k_{12}=15.00$ & \\
& $k_{7}=15.00, k_{8}=15.00$ & \\
& $k_{9}=14.29, k_{10}=14.79$ & \\
& $k_{11}=14.70, k_{12}=15.00$ &
\end{tabular}

of the fitness function with number of iterations is shown in figure 4. Meanwhile, the variations of backstepping control parameters with respect to the number of iterations are shown in figure 5 . For the position controller, the best fitness value is 0.1385 appeared in iteration number 7 , and the optimal parameters are $k_{7}=15.00, k_{8}=15.00, k_{9}=14.29, k_{10}=14.79, k_{11}=14.70$ and $k_{12}=15.00$. The variation of the fitness function with number of iterations is shown in figure 6 . Meanwhile, the variations of backstepping control parameters with respect to the number of iterations are shown in figure 7. As can be seen for both control parameters optimization, through about 20 iterations, the GSA method can prompt convergence and obtain good fitness value.

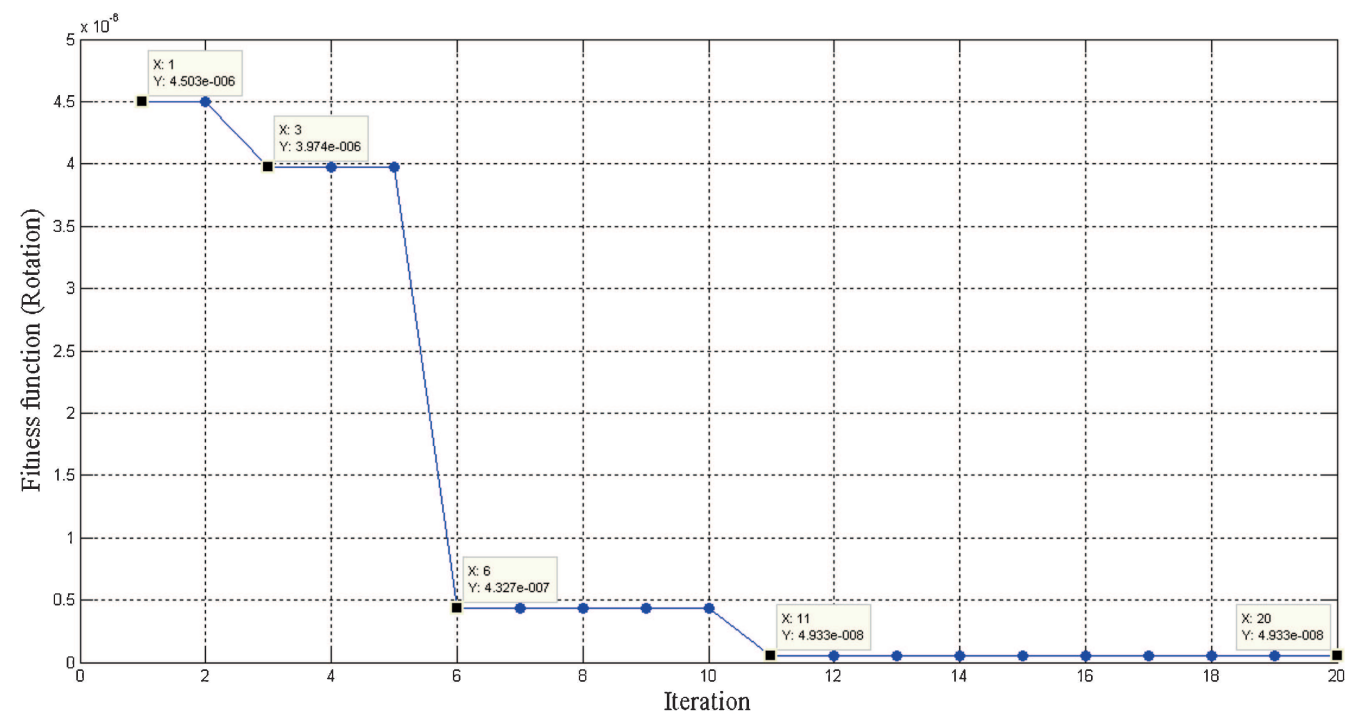

Figure 4. The convergence of fitness function for rotation controller with number of iterations. 


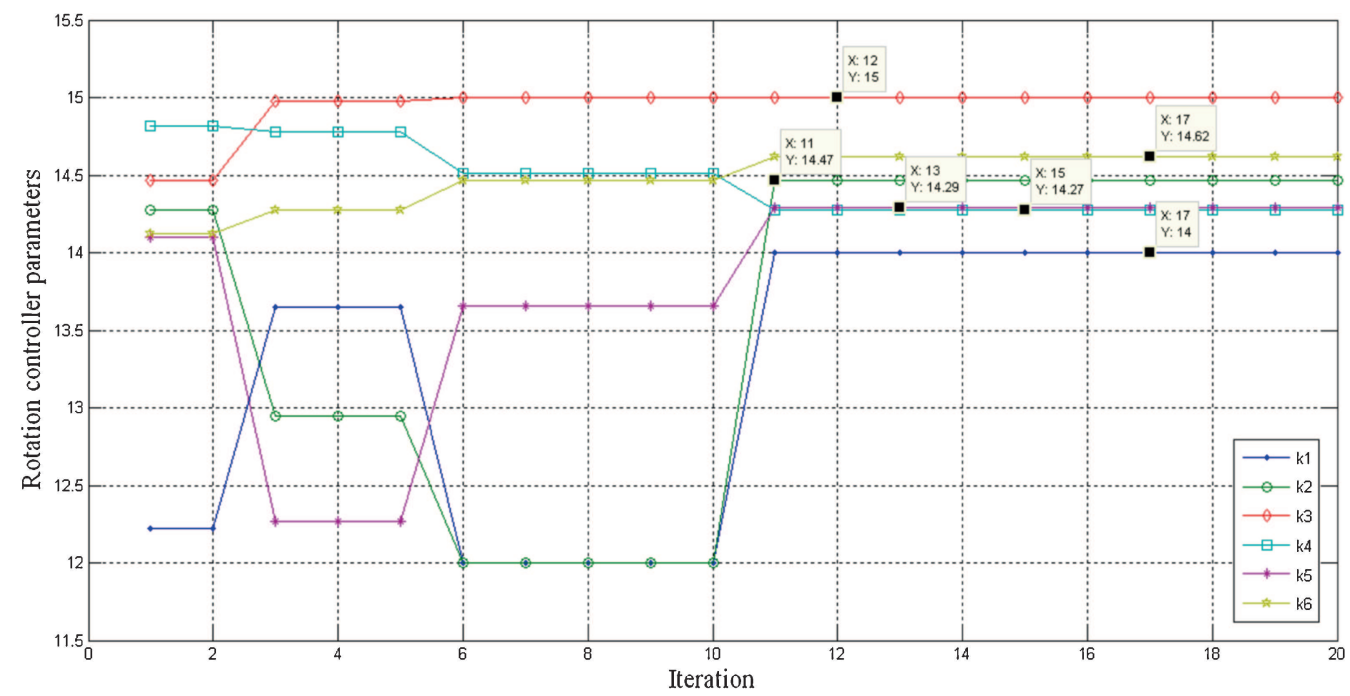

Figure 5. The variations of rotation controller parameters versus number of iterations.

To explore the effectiveness of the proposed robust optimal backstepping controller, two simulation experiments have been performed on the quadrotor. In the first experiment, the simulation results of the proposed controller in a stabilizing problem are given. In the second, the performance of the scheme is investigated in trajectory tracking problem. Two test conditions are provided, which are the nominal case (Case 1) and the external disturbance case (Case 2). The external disturbance is generated as forces produced by wind that is modeled using Dryden Wind-Gust model (Waslander \& Wang 2009).

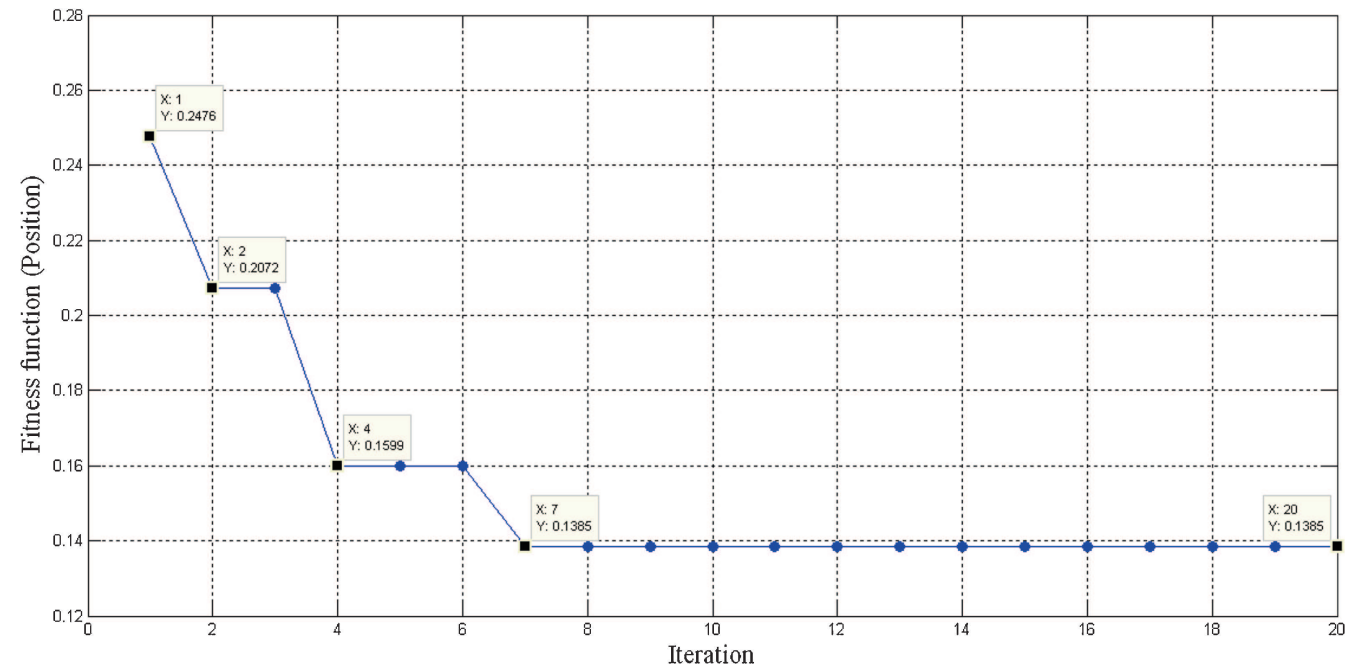

Figure 6. The convergence of fitness function for position controller with number of iterations. 


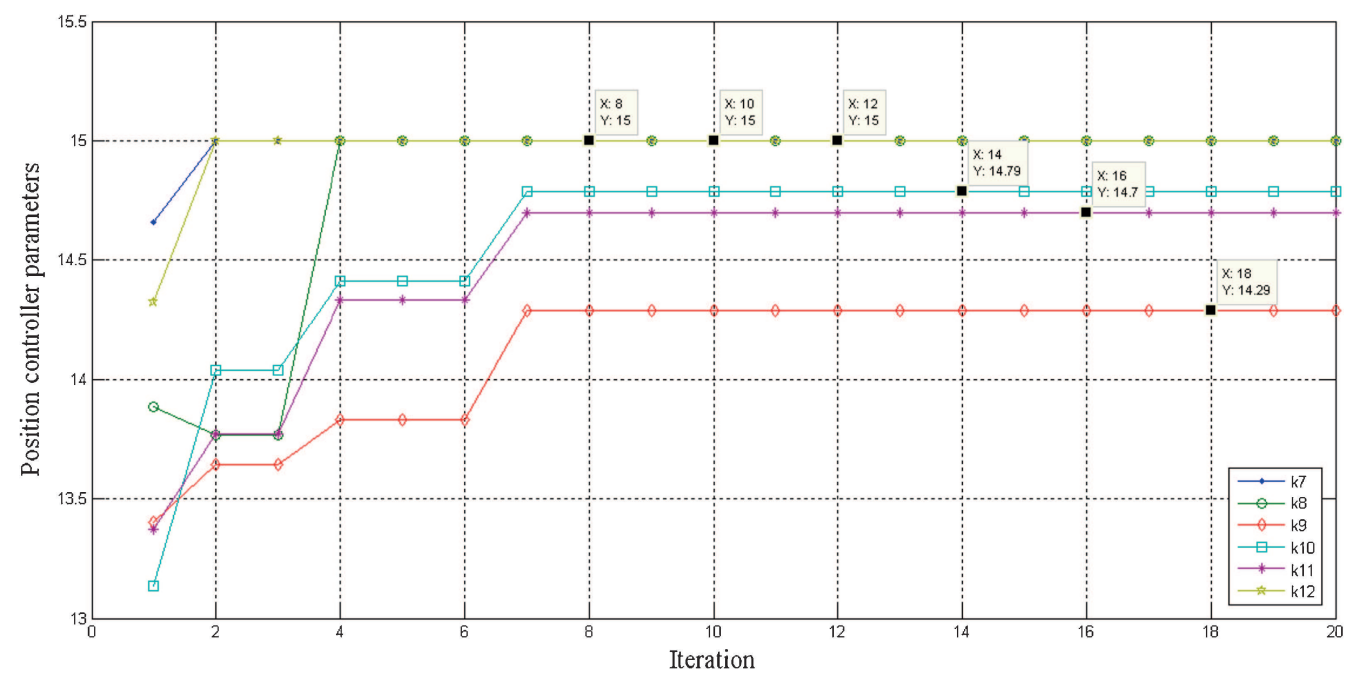

Figure 7. The variations of position controller parameters versus number of iterations.

\subsection{Simulation experiment 1: stabilizing problem}

In this simulation experiment, the control objective is to regulate a quadrotor at a certain desired altitude/attitude, such that the quadrotor can hover at a fixed point. The desired altitude/attitude is given by $x_{i d}=\left[z_{d}, \phi_{d}, \theta_{d}, \psi_{d}\right]=[5,0,0,0]^{T}$. The initial states are given by $z=5, \phi=0.2$,
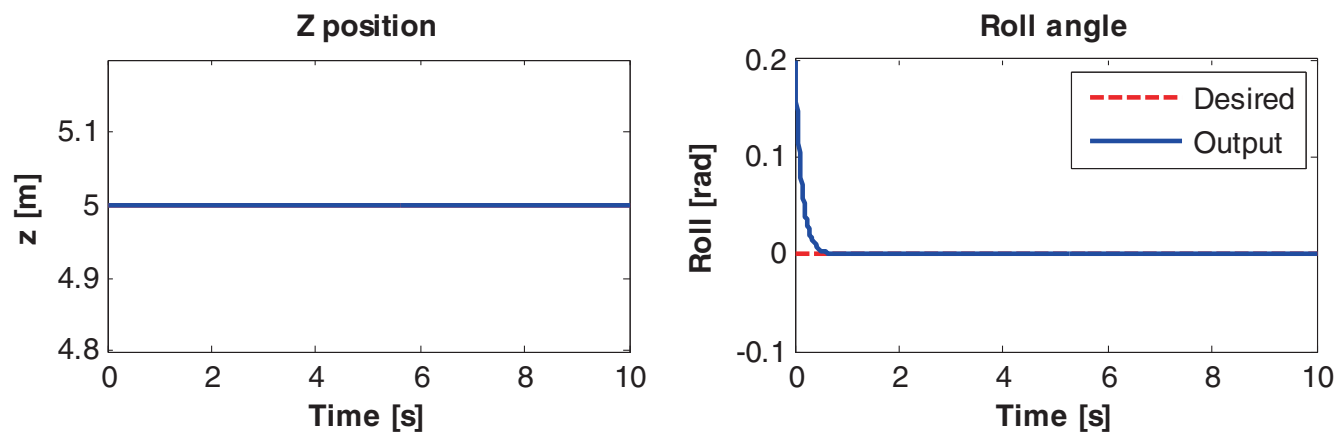

Pitch angle
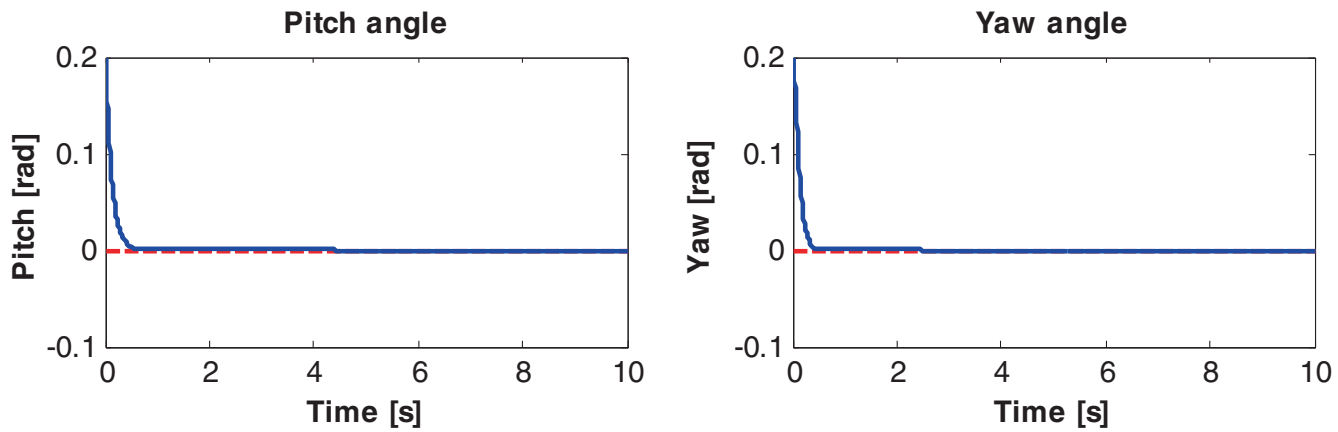

Figure 8. Altitude/attitude stabilization of the hovering quadrotor using $\mathrm{OBC}$ at Case 1. 

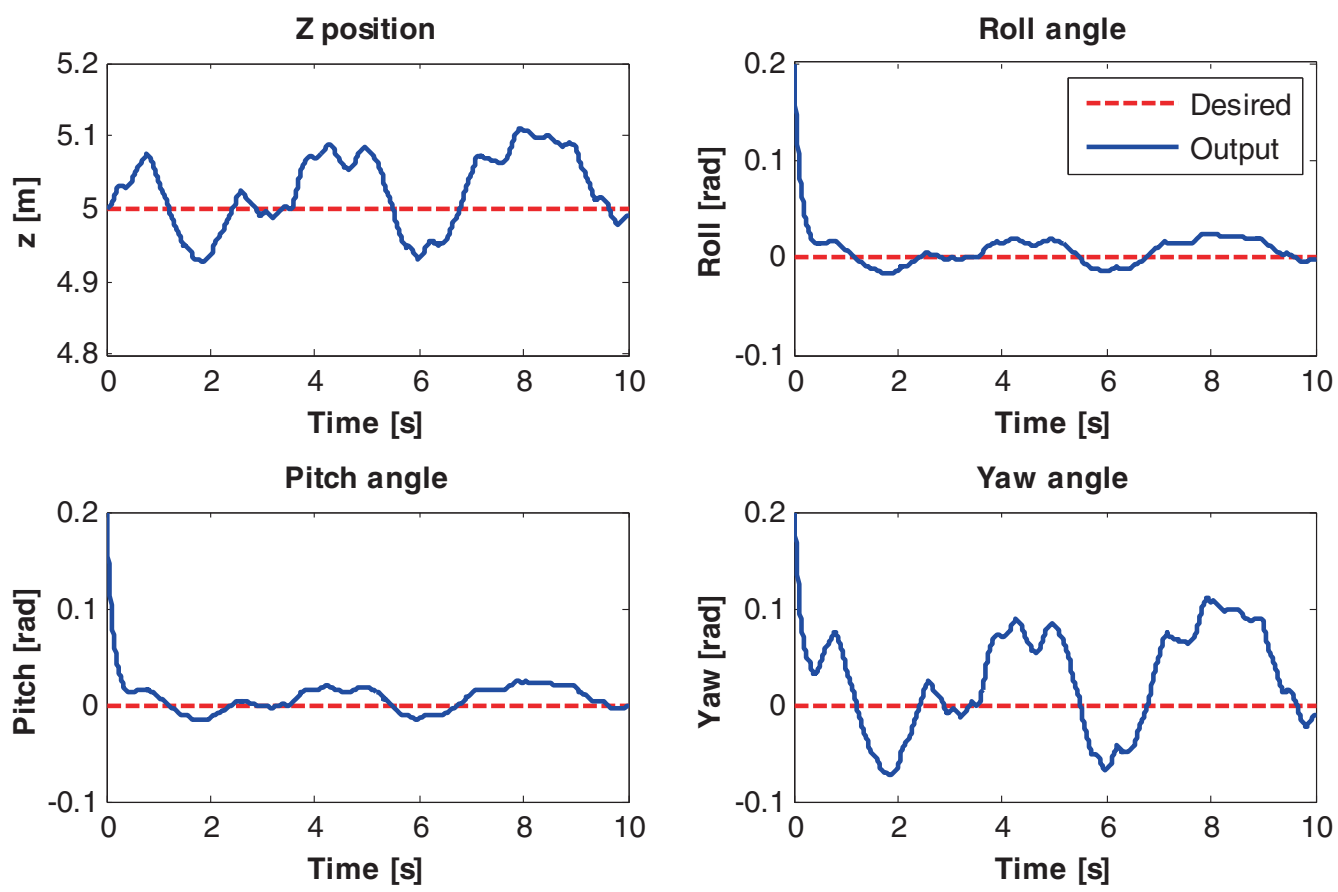

Figure 9. Altitude/attitude stabilization of the hovering quadrotor using $\mathrm{OBC}$ at Case 2.
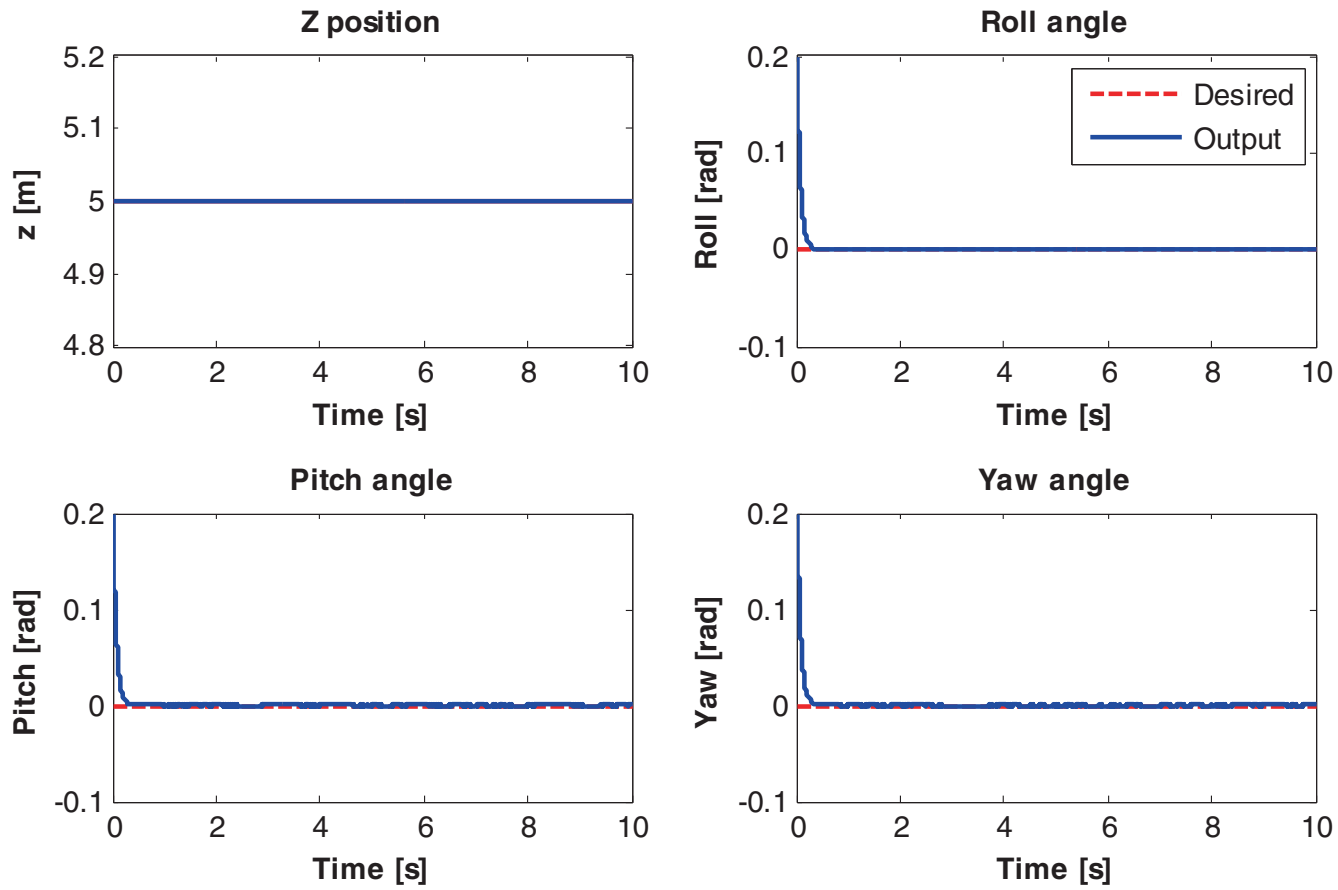

Figure 10. Altitude/attitude stabilization of the hovering quadrotor using ROBC at Case 1. 

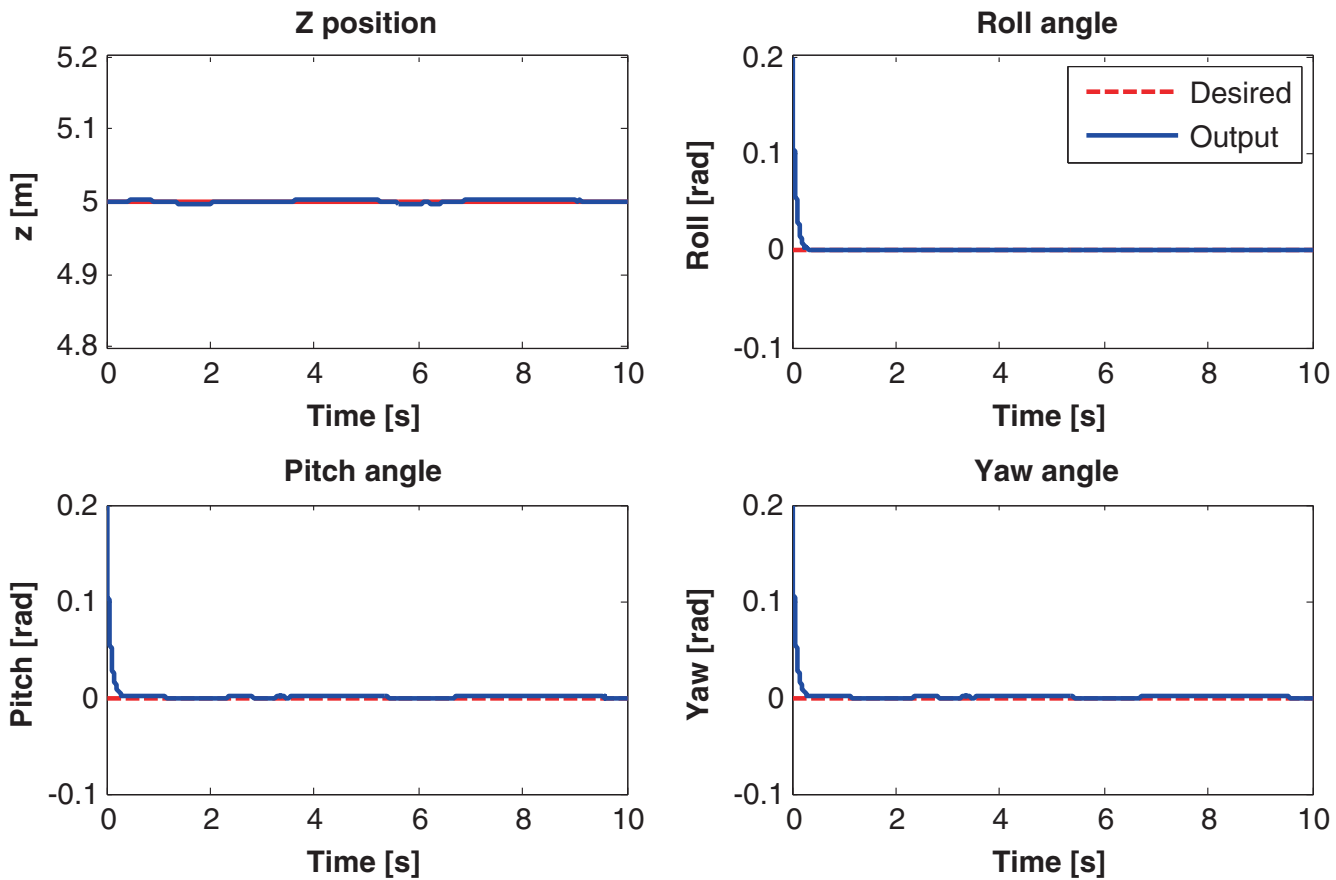

Figure 11. Altitude/attitude stabilization of the hovering quadrotor using ROBC at Case 2 .
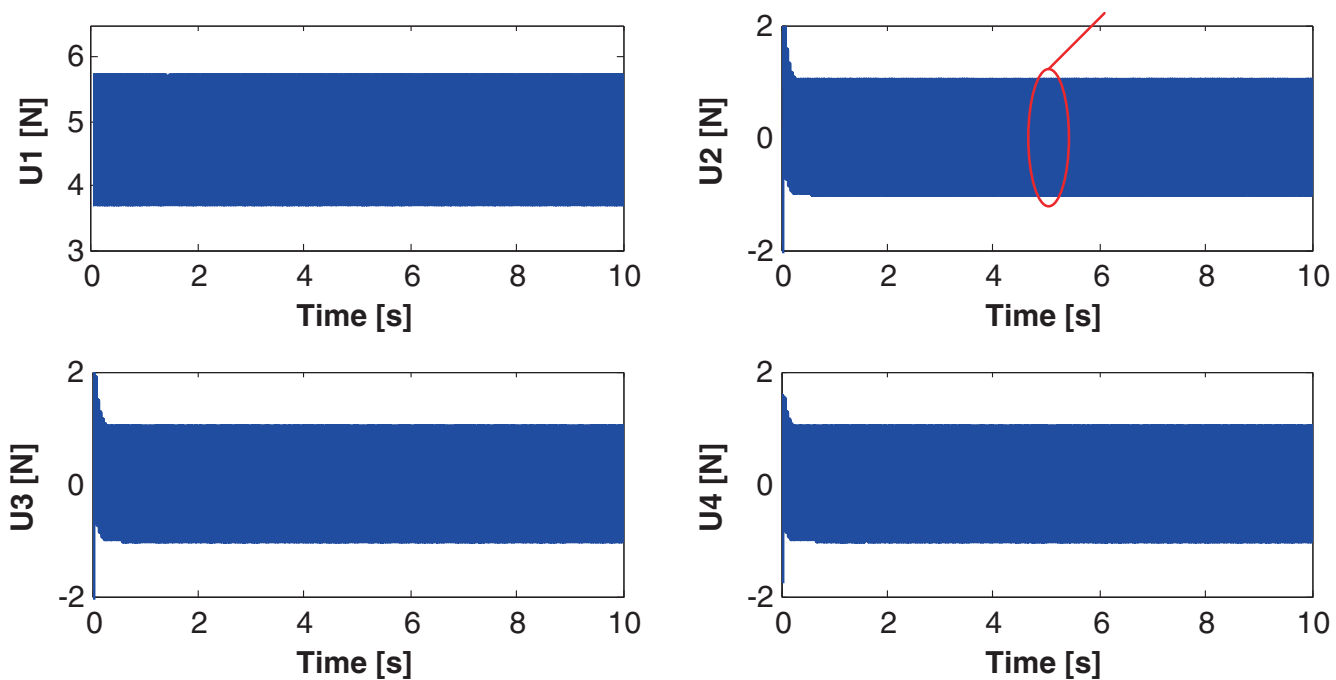

Figure 12. Control inputs of ROBC with sign function. 

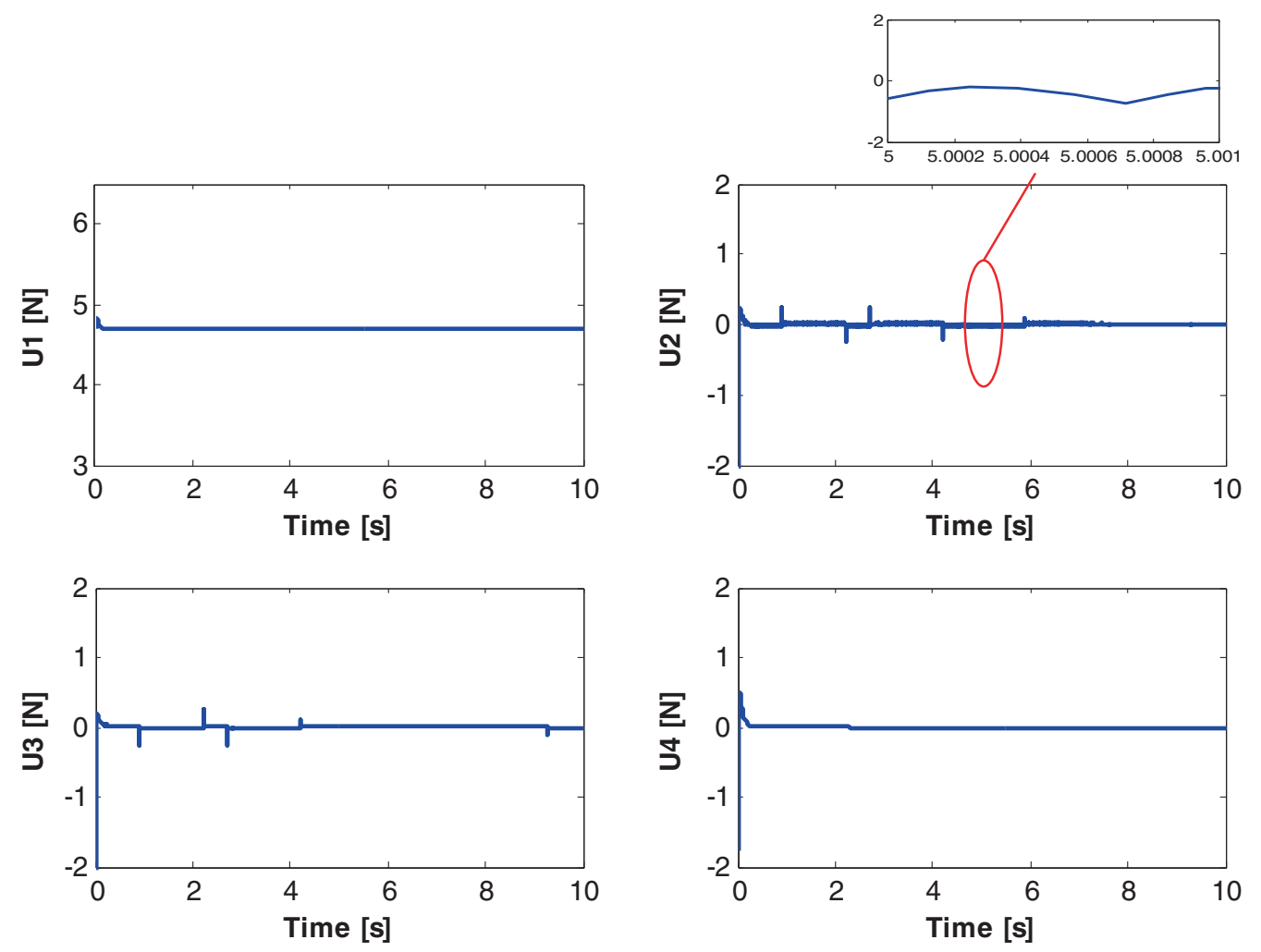

Figure 13. Control inputs of $\mathrm{ROBC}$ with saturation function.

$\theta=0.2$ and $\psi=0.2$. In the simulation, first, the OBC system is considered. The simulation results of the $\mathrm{OBC}$ system for stabilizing a quadrotor at Case 1 and Case 2 are depicted in figures 8 and 9, respectively. From the simulation results, the OBC system is able to stabilize the quadrotor in hover mode at Case 1 . However, the degenerate performance response shown in figure 9 is resulted under the occurrence of external disturbance. Under the same simulation cases, the ROBC system is simulated. The simulation results for stabilizing a quadrotor at Case 1 and Case 2 are depicted in figures 10 and 11, respectively. From the simulation results, the robust control performance of the proposed ROBC system in the quadrotor stabilization can be observed. It can be clearly seen that the altitude/attitude of the quadrotor can be maintained at the desired altitude/attitude, that is, the hovering flight is stable even if the external disturbance is exerted as shown in figure 11. As aforementioned, the using of sign switching function will cause undesirable phenomenon called chatter in the control input as shown in figure 12 . The effective way to counter this problem is by utilizing the saturation function and the result can be seen in figure 13 .

\subsection{Simulation experiment 2: trajectory tracking problem}

In this simulation experiment, the control objective is to ensure the quadrotor can effectively track the desired reference trajectory. The helical trajectory is adopted to test the trajectory 


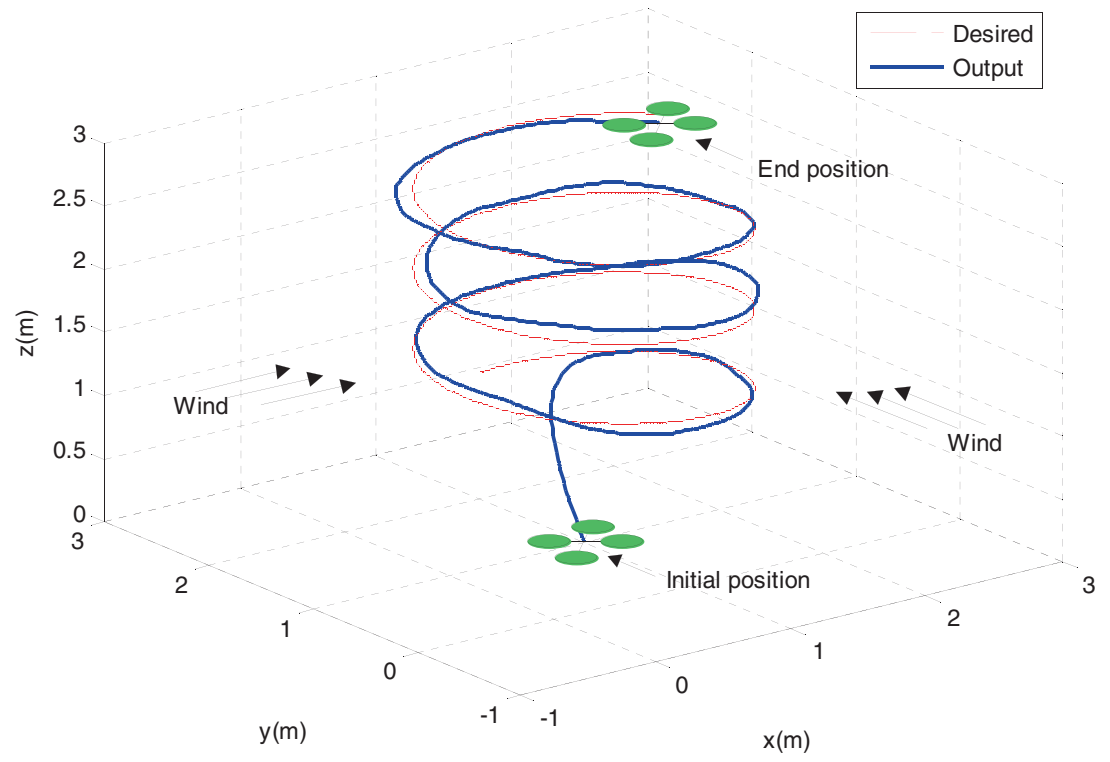

Figure 14. Helical trajectory tracking response using $\mathrm{OBC}$ at Case 2.

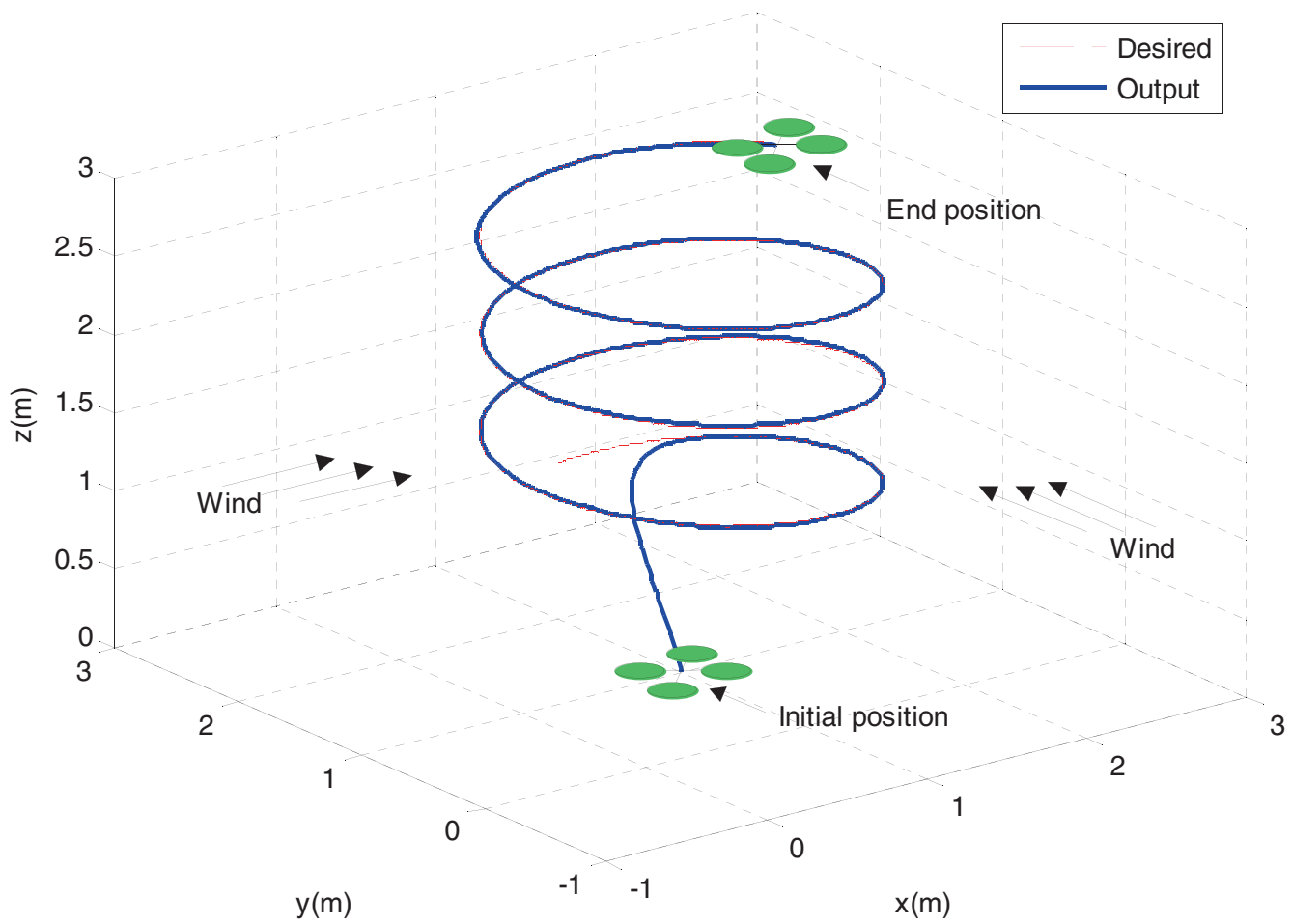

Figure 15. Helical trajectory tracking response using ROBC at Case 2. 
tracking capability of the quadrotor by the OBC and ROBC system. The desired trajectory is generated using the following command:

$$
\left\{\begin{array}{l}
x_{d}=\sin (t), \\
y_{d}=\cos (t), \\
z_{d}=1+0.2 t .
\end{array}\right.
$$

The initial state of the quadrotor is set to be $\left[x_{0}, y_{0}, z_{0}\right]=[1,1,0] \mathrm{m}$. The simulation results of helical trajectory tracking for both $\mathrm{OBC}$ and $\mathrm{ROBC}$ approaches under the occurrence of external disturbance are respectively shown in figures 14 and 15. As it can be seen, the proposed control scheme can track the desired reference trajectory accurately despite external disturbance. It is obvious that the ROBC can give small tracking error and good tracking performance compared to $\mathrm{OBC}$.

\section{Conclusions}

In this paper, the application of a robust controller for stabilization and trajectory tracking of a quadrotor helicopter perturbed by external disturbances is successfully demonstrated. First, the mathematical model of the quadrotor is introduced. Then, the proposed robust control system, which comprises a backstepping and a switching function, is developed. The backstepping control design is derived based on Lyapunov function, so that the stability of the system can be guaranteed, while switching function is used to attenuate the effects caused by disturbances. GSA has been utilized to determine the controller parameters. Finally, the proposed control scheme is applied to autonomous quadrotor helicopter. Simulation results show that high-precision transient and tracking response can be achieved by using the proposed control system.

\section{References}

Baek S J, Lee D J, Park J H and Chong K T 2013 Design of lateral Fuzzy-PI controller for unmanned quadrotor robot. J. Inst. Control, Robotics Syst. 19: 164-170

Bolandi H, Rezaei M, Mohsenipour R, Nemati H and Smailzadeh S 2013 Attitude control of a Quadrotor with optimized PID controller. Intelligent Control Autom. 4: 335-342

Bošković D M and Krstić M 2002 Backstepping control of chemical tubular reactors. Comp.Chem. Eng. 26: 1077-1085

Bouadi H, Bouchoucha M and Tadjine M 2007 Modelling and stabilizing control laws design based on sliding mode for an UAV Type-Quadrotor. Eng. Lett. 15: 342-347

Bouadi H, Bouchoucha M and Tadjine M 2008 Sliding mode control based on backstepping approach for an UAV type-quadrotor. Int. J. Appl. Math. Comp. Sci. 4: 12-17

Castillo P, Lozano R and Dzul A 2005 Stabilization of a mini rotorcraft with four rotors. IEEE Control Syst. Mag. 25: 45-55

Chiu C H, Peng Y F and Lin Y W 2011 Intelligent backstepping control for wheeled inverted pendulum. Expert Syst. Appl. 38: 3364-3371

De Moura Oliveira P, Pires E S and Novais P 2013 Gravitational search algorithm design of Posicast PID control systems. Soft Computing Models in Industrial and Environmental Applications (pp. 191-199): Springer

Erginer B and Altug E 2012 Design and implementation of a hybrid fuzzy logic controller for a quadrotor VTOL vehicle. Int. J. Control, Autom. Syst. 10: 61-70 
Guisser M H and Medromi H 2009 A high gain observer and sliding mode controller for an autonomous quadrotor helicopter. Int. J. Intell. Control Syst. 14: 204-212

Hu Q, Xu L and Zhang A 2012 Adaptive backstepping trajectory tracking control of robot manipulator. J. Franklin Inst. 349: 1087-1105

Hwang J H, Hwang S, Hong S K and Yoo M G 2012 Attitude stabilization performance improvement of the quadrotor flying robot. J. Inst. Control Robot. Syst. 18: 608-611

Jafari H, Zareh M, Roshanian J and Nikkhah A 2010 An optimal guidance law applied to quadrotor using LQR method. Trans. Japan Soc. Aeronaut. Space Sci. 53: 32-39

Jiang Y, Hu Q and Ma G 2010 Adaptive backstepping fault-tolerant control for flexible spacecraft with unknown bounded disturbances and actuator failures. ISA Trans. 49: 57-69

Junior J C V, De Paula J C, Leandro G V and Bonfim M C 2013 Stability control of a quad-rotor using a PID controller. Brazilian J. Instrum. Control 1: 15-20

Kim H M, Park S H, Song J H and Kim J S 2010 Robust position control of electro-hydraulic actuator systems using the adaptive back-stepping control scheme. Proc. Inst. Mech. Eng. I: J. Syst. Control Eng. 224: 737-746

Kristiansen R, Nicklasson P J and Gravdahl J T 2009 Satellite attitude control by quaternion-based backstepping. IEEE Trans. Control Syst. Technol. 17: 227-232

Krstic M, Kanellakopoulos I and Kokotovic P 1995 Nonlinear and adaptive control design, (Vol. 222): Wiley New York

Lee D, Ha C and Zuo Z 2013 Backstepping control of quadrotor-type UAVs and its application to teleoperation over the Internet. 12th International Conference on Intelligent Autonomous Systems, Jeju Island, vol. 194, pp. 217-225

Madani T and Benallegue A 2006 Backstepping control for a quadrotor helicopter. IEEE/RSJ International Conference on Intelligent Robots and Systems, pp. 3255-3260

Mokhtari A, M'Sirdi N K, Meghriche K and Belaidi A 2006 Feedback linearization and linear observer for a quadrotor unmanned aerial vehicle. Adv. Robot. 20: 71-91

Mukherjee P and Waslander S L 2012 Direct adaptive feedback linearization for quadrotor control. AIAA Guidance, Navigation, and Control Conference

Nuchkrua T and Parnichkun M 2012 Identification and optimal control of Quadrotor. Thammasat Int. J. Sci. Technol. 17: 36

Olfati-Saber R 2000 Nonlinear control of underactuated mechanical systems with application to robotics and aerospace vehicle. Ph.D. thesis, Massachusetts Institute of Technology

Rashedi E, Nezamabadi-Pour H and Saryazdi S 2009 GSA: a gravitational search algorithm. Information Sci. 179: 2232-2248

Regula G and Lantos B 2011 Backstepping based control design with state estimation and path tracking to an indoor quadrotor helicopter. Electr. Eng. Comput. Sci. 53: 151-161

Santos M, López V and Morata F 2010 Intelligent fuzzy controller of a quadrotor. IEEE International Conference on Intelligent Systems and Knowledge Engineering, pp. 141-146

Shen Q, Jiang B and Cocquempot V 2013 Adaptive fault-tolerant backstepping control against actuator gain faults and its applications to an aircraft longitudinal motion dynamics. Int. J. Robust Nonlinear Control 23: $1753-1779$

Sumantri B, Uchiyama N, Sano S and Kawabata Y 2013 Robust tracking control of a quad-rotor. Helicopter utilizing sliding mode control with a nonlinear sliding surface. J. Syst. Des. Dyn. 7: 226-241

Sun W, Gao H and Kaynak O 2013 Adaptive backstepping control for active suspension systems with hard constraints. IEEE/ASME Trans. Mechatronics 18: 1072-1079

Voos $\mathrm{H} 2009$ Nonlinear control of a quadrotor micro-UAV using feedback-linearization. IEEE International Conference on Mechatronics, pp. 1-6

Waslander S L and Wang C 2009 Wind disturbance estimation and rejection for quadrotor position control. AIAA Infotech@ Aerospace Conference and AIAA Unmanned... Unlimited Conference, Seattle, WA

Xu J X, Guo Z Q and Lee T H 2012 Synthesized design of a fuzzy logic controller for an underactuated unicycle. Fuzzy Sets Syst. 207: 77-93 
Yang J H and Yang K S 2012 An adaptive variable structure control scheme for underactuated mechanical manipulators. Math. Problems Eng. 2012

Yih C C 2013 Sliding mode control for swing-up and stabilization of the cart-pole underactuated system. Asian J. Control 15: 1201-1214

Yu R, Zhu Q, Xia G and Liu Z 2012 Sliding mode tracking control of an underactuated surface vessel. IET Control Theory Appl. 6: 461-466

Zhang Y, Zhao W, Lu T and Li J 2013 The attitude control of the four-rotor unmanned helicopter based on feedback linearization control. WSEAS Trans. Syst. 12: 229-239

Zilic T, Kasac J, Situm Z and Essert M 2013 Simultaneous stabilization and trajectory tracking of underactuated mechanical systems with included actuators dynamics. Multibody Syst. Dyn. 29: 1-19 